\title{
Ein eingespieltes Team
}

\section{Das Interview führte: Julia Rippstein}

Redaktorin Print Online

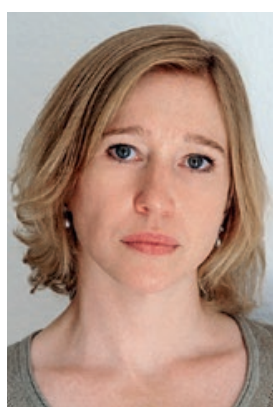

Nadja Pecinska

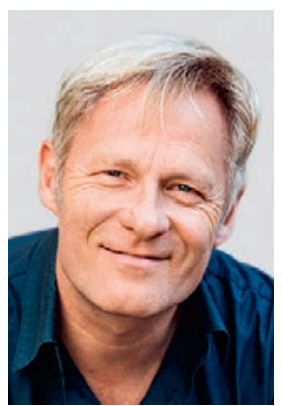

Christian Heller

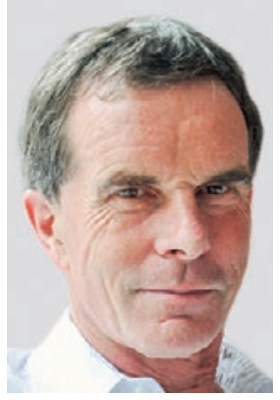

Reto Krapf

Gleichzeitig mit dem Veröffentlichungsdatum dieser SÄZ erscheint die 25. Folge des vom Schweizerischen Ärzteverlag EMH produzierten Podcast «EMH Journal Club». Die Projektinitiantin Dr. med. Nadja Pecinska, Managing Editorin von Primary and Hospital Care, ist mit der Entwicklung des neuen Formats sehr zufrieden.

Nadja Pecinska, heute, am 21. Oktober stellen Sie bereits den 25. Podcast ins Netz - ein kleines Jubiläum. Wie geht es Ihnen dabei?

Sehr gut, danke. Nun, da wir ja doch schon etwas Übung haben und ein eingespieltes Team sind, läuft die Produktion einwandfrei. Im Gegensatz zu unserem Sprecher Christian Heller bin ich ja kein Profi. Aber ich habe schon sehr viel dazu gelernt! Es geht da vor allem um das flüssige Sprechen, die richtigen Betonungen und die Konstanz der Stimmfarbe. Zehn Monate nach dem Start des Podcasts bin ich nach wie vor voll motiviert für dieses Projekt und überzeugt davon. Die Zahlen bekräftigen meinen Eindruck: Wir haben jetzt fast 3000 Abonnenten. Dies freut mich sehr, denn es heisst, es gibt 3000 Personen, die sich aktiv für unser Produkt interessieren.

\section{Wissen Sie, wer Ihre «Follower» sind?}

Nicht genau. Es sind aber sicherlich mehrheitlich Ärztinnen und Ärzte sowie Forschende, denn das Format des «Journal Club» ist explizit an ein Fachpublikum gerichtet. Es entspricht dem der EMH-Zeitschriften. Die meisten Hörerinnen und Hörer sind aus der Schweiz, ein kleiner Teil aus Deutschland und aus anderen Ländern. Wir hoffen jedoch, dass wir unsere Hörerschaft jenseits der Grenzen vergrössern können. In Deutschland und Österreich gäbe es wohl noch ein grosses Potenzial.

\section{Wie könnten Sie dieses Publikum «ennet der Grenze» besser erreichen?}

Es ist nicht einfach. Wir gelangen zwar gut an die Leserschaft der EMH-Zeitschriften, aber die Medizin- und Wissenschaftsinteressierten, die unsere Zeitungen nicht erhalten, sind schwer darüber zu informieren, dass es diesen Podcast gibt. Mund-zu-Mund-Propaganda könnte da etwas bewegen. Beispielsweise, wenn unsere Abonnentinnen und Abonnenten ihren deutschen resp. österreichischen Kolleginnen und Kollegen den Tipp weitergeben würden - darüber würde ich mich natürlich sehr freuen.
Während des Lockdowns wurden sieben ExtraPodcasts zu COVID-19 veröffentlicht. Wie sind diese bei der Hörerschaft angekommen?

Die Extra-Podcasts, welche die neuesten COVID-19-Studien zusammenfassten, wurden rege gehört, was uns sehr gefreut und den Zusatzaufwand sozusagen «entschädigt» hat. Die Serie «EMH Journal Club COVID-19 Extra» ist nun beendet, aber auch die "normalen" Folgen, die es ab dem 1. Juli wieder in der gewohnten zweiwöchentlichen Frequenz gibt, beinhalten jeweils zwei bis drei COVID-19-Studien. Diese werden wie die anderen Studien von Professor Dr. Reto Krapf, Autor der Rubrik «Kurz und bündig» vom Swiss Medical Forum, zusammengefasst und im Podcast kommentiert. Der Podcast basiert ja auf dieser Rubrik. Aber das Schöne am Podcast-Format ist, dass alle Folgen zu jeder Zeit abrufbar sind, die Extra-Podcasts zu COVID-19 können also immer und jederzeit gehört werden.

\section{Noch ein Wort über die Zukunft?}

Wir hoffen, dass es sich so weiterentwickelt und dass weitere Podcasts in den nächsten Jahren dazukommen, sei es zu anderen Themen, für andere Zielgruppen oder in anderen Sprachen, insbesondere auf Französisch. Kurzfristiger setzen wir uns als Ziel, ein noch grösseres Publikum innerhalb des deutschsprachigen Raums anzusprechen.

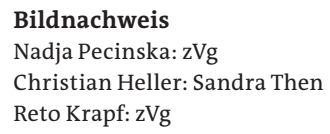

Unter emh.ch/podcast sind alle Podcasts jederzeit abrufbar. Der Podcast «EMH Journal Club» kann überall dort abonniert werden, wo es Podcasts gibt (Apple Podcasts, Google Podcasts, Spotify etc). Mehr Infos zur Rubrik "Kurz und bündig» von Prof. Dr. med. Reto Krapf: https:// medicalforum.ch/online-magazine

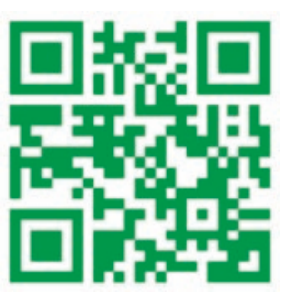

